\title{
Salvia miltiorrhiza Injection Ameliorates Renal Damage Induced by Lead Exposure in Mice
}

\author{
Lei Li, ${ }^{1,2}$ Yuanyuan Zhang, ${ }^{1}$ Juanjuan Ma, ${ }^{1}$ Weichong Dong, ${ }^{3}$ \\ Qiongtao Song, ${ }^{1}$ Jianping Zhang, ${ }^{1}$ and Li Chu ${ }^{1}$ \\ ${ }^{1}$ Department of Pharmacology, School of Basic Medicine, Hebei Medical University, 326 Xinshi South Road, \\ Shijiazhuang, Hebei 050091, China \\ ${ }^{2}$ Department of Internal Medicine, Baoding First Hospital of Traditional Chinese Medicine, Yuhua Western Road, \\ Baoding, Hebei 071000, China \\ ${ }^{3}$ Department of Pharmaceutical Analysis, School of Pharmacy, Hebei Medical University, 361 Zhongshan East Road, \\ Shijiazhuang, Hebei 050017, China
}

Correspondence should be addressed to Jianping Zhang; zhangjianping14@126.com and Li Chu; chuli0614@126.com

Received 12 December 2013; Accepted 1 January 2014; Published 13 February 2014

Academic Editors: M. Galuppo, M. Makishima, and J. B. T. Rocha

Copyright (C) 2014 Lei Li et al. This is an open access article distributed under the Creative Commons Attribution License, which permits unrestricted use, distribution, and reproduction in any medium, provided the original work is properly cited.

\begin{abstract}
Exposure to lead $(\mathrm{Pb})$ can induce kidney injury and our recent studies have found that Salvia miltiorrhiza (SM) injection, a traditional Chinese medicine, could protect against the organ injury induced by iron overload. This study was designed to investigate the protective effects of SM injection on nephrotoxicity induced by $\mathrm{Pb}$ acetate in mice and to elucidate the potential mechanism(s). Healthy male mice were randomly divided into four groups: control, Pb, low-dose Salvia miltiorrhiza (L-SM), and high-dose Salvia miltiorrhiza (H-SM). SM injection dose dependently reduced the $\mathrm{Pb}$ accumulation in the kidney, decreased kidney coefficients, and ameliorated renal structure and function from the morphology analysis. Meanwhile, SM administration downregulated serum levels of blood urea nitrogen (BUN) and creatinine (CR), decreased malondialdehyde (MAD) content, and increased activities of super oxide dismutase (SOD) and glutathione peroxidase (GSH-Px) in the kidney homogenate. Moreover, SM injection reduced the level of renal apoptosis by immunohistochemical staining analysis. Our findings implicate the therapeutic potential of SM injection for $\mathrm{Pb}$-induced nephrotoxicity, which were at least partly due to the decrease of $\mathrm{Pb}$ accumulation, inhibition of lipid peroxidation, and suppression of renal apoptosis. These results provided preliminary experimental support for Danshen as a therapeutic drug for $\mathrm{Pb}$ poisoning diseases.
\end{abstract}

\section{Introduction}

Lead $(\mathrm{Pb})$ is a common environmental contaminant and excessive exposure to it may cause chronic or acute nephrotoxic effects. Acute $\mathrm{Pb}$ nephropathy, mainly reported in children, is characterized functionally by a generalized deficit of tubular transport mechanisms (Fanconi syndrome) and morphologically by the appearance of degenerative changes in the tubular epithelium and nuclear inclusion bodies containing $\mathrm{Pb}$ protein complexes [1]. $\mathrm{Pb}$ poisoning mainly inhibits cell enzymes that contained thiol and leads to the body's biochemical and physiological dysfunction, causing small artery spasm and capillary endothelial cell injury, changing the properties of the normal erythrocyte membrane, inhibiting muscle phosphocreatine resynthesis, and so forth. Thus this gives rise to a series of pathological changes, wherein the nervous system, the kidneys, and hematopoietic system are more remarkable.

Clinical trials and epidemiological studies have demonstrated that lead poisoning can cause kidney damage. Currently, chelating agents such as disodium edetate calcium $\left(\mathrm{EDTANa}_{2} \mathrm{Ca}\right)$ and sodium dimercaptosuccinate (NaDMS) are commonly used in clinical to treat lead poisoning. Chelating agents form an insoluble complex with $\mathrm{Pb}$ for removal from the $\mathrm{Pb}$-burdened tissue, but they are incapable of removing metal from intracellular sites and may cause 
redistribution of the toxic metal, essential metal loss, and liver or renal dysfunction [2].

Salvia miltiorrhiza (SM), commonly known as Danshen, has been used for hundreds of years in China and other countries. SM injection, the aqueous extracts of SM, has been widely and successfully used in the treatment of cardiovascular diseases and renal failure [3]. Recent studies have highlighted the diversity of potential effects of SM in attenuating microcirculatory disturbances, including antioxidation, inhibition of apoptosis, and amelioration of injury to target organs such as the kidney [4]. And our recent studies have found that SM injection could protect against cardiac damage, liver injury, hepatic fibrosis, and kidney injury induced by acute or chronic iron overload [5-8]. So we deduced that SM may have a protective effect on the renal damage induced by divalent heavy metal poisoning.

Voltage-dependent calcium channels found in a variety of cells are blocked by different metal cations. $\mathrm{Pb}^{2+}$ has been shown to perturb $\mathrm{Ca}^{2+}$-mediated cellular processes [9]. $\mathrm{Pb}^{2+}$ mimics $\mathrm{Ca}^{2+}$ in many biochemical and physiological processes and also competes with $\mathrm{Ca}^{2+}$ for membrane channels to gain entry into the cell [10]. Our recent study indicated that calcium channel blockers (CCBs) verapamil and nimodipine may have protective effects on nephrotoxicity induced by $\mathrm{Pb}$ acetate in mice [11]. Consequently, inhibiting $\mathrm{Ca}^{2+}$ channels has become a potential therapeutic approach for reducing kidney injury induced by $\mathrm{Pb}$ exposure. According to previous research findings, salvianolic acid $B$, one of the main active components in SM injection, has the capability of reducing the production of oxygen free radicals [12] and inhibiting $\mathrm{Ca}^{2+}$ channels [13]. In addition to the antioxidation and antiapoptosis, SM may act as a CCB to fight against renal injury induced by $\mathrm{Pb}$ poisoning.

In view of the importance of $\mathrm{Pb}$ intoxication in the pathological process of renal disease, it needs new drugs to treat $\mathrm{Pb}$ poisoning. Therefore, this study investigated the $\mathrm{SM}$ injection to protect against $\mathrm{Pb}$ nephrotoxicity in mice and the potential mechanisms.

\section{Materials and Methods}

2.1. Animals and Drugs. Eighty male Kunming mice weighing $20.0 \pm 3.0 \mathrm{~g}$ were purchased from the Experimental Animal Center, Hebei Medical University, and housed in plastic cages with well-ventilated stainless steel grid tops at controlled temperature with a $12 \mathrm{~h}$ light-dark cycle. Animals were allowed to access normal chow and drinking water ad libitum. All animal handling procedures were in accordance with the Guidelines of Animal Experiments from the Committee of Medical Ethics, Ministry of Health of China, and experiments were approved by the Ethics Committee for Animal Experiments of Hebei Medical University (approval number: HEBMU-2011-09, approval date: September 07, 2011).

SM injection, which was approved by the State Food and Drug Administration (approval number: Z32020161), was obtained from Shenlong Pharmaceutical Co., Ltd. (Jiangsu, China, batch number: 11040314). Pb acetate (analytically pure) was obtained from Kangpuhuiwei Technology Co., Ltd. (Beijing, China). The reference substances (purity $\geq$ 98\%) for HPLC-UV: danshensu, protocatechuic aldehyde, and salvianolic acid B were purchased from the Tauto Biotech Co., Ltd. (Shanghai, China). Unless otherwise stated, other chemical reagents were obtained from Sigma-Adrich Inc. (St. Louis, MO).

2.2. Experimental Design. After one week of acclimation, all mice were randomly divided into four groups $(n=20$ in each group): control, $\mathrm{Pb}$, low-dose Salvia miltiorrhiza (L$\mathrm{SM})$, and high-dose Salvia miltiorrhiza (H-SM). Mice of the L-SM and H-SM groups were given i.p. injections of $\mathrm{SM}$ at 3 and $6 \mathrm{~g} \cdot \mathrm{kg}^{-1}$ per day (calculated according to the content of SM, $1.5 \mathrm{~g} \cdot \mathrm{mL}^{-1}$ ), respectively. Meanwhile, mice of the control group and $\mathrm{Pb}$ group received isovolumic saline by the same route. Mice of the $\mathrm{Pb}, \mathrm{L}-\mathrm{SM}$, and $\mathrm{H}$-SM groups were intraperitoneally (i.p.) injected with $\mathrm{Pb}$ acetate $40 \mathrm{mg} \cdot \mathrm{kg}^{-1}$ per day four hours later on the same day. Furthermore, the normal saline (NS) was used to dilute the SM before injection. The entire experimental period lasted for 10 days, and all mice were maintained on normal chow and drinking water ad libitum. Food intake and activities of all mice were observed carefully every day, and body weight was measured at the beginning and end of the experiment. After recording the final body weight on the 10th day of the experiment, each animal was anesthetized with sodium pentobarbital $\left(50 \mathrm{mg} \cdot \mathrm{kg}^{-1}\right)$. Blood was taken from femoral arteries and the serum was separated for further analysis. Subsequently, the kidneys were quickly excised and stored in the $4 \%$ paraformaldehyde solution or cryopreserved at $-80^{\circ} \mathrm{C}$.

2.3. Main Constituent Analysis of SM Injection. The main constituents of the SM injection were detected by high performance liquid chromatography with ultraviolet (HPLCUV) and the chromatograms were presented in Figure 1. The conditions and procedures have been described previously [5].

2.4. Determination of Kidney Coefficients. Kidneys were removed and weighed using an electronic balance. The kidney coefficients were calculated as the kidney to body mass ratio $\times 100$.

2.5. Tissues $\mathrm{Pb}$ Measurement. $\mathrm{Pb}$ concentrations in kidney homogenate were analyzed by flame atomic absorption spectroscopy (FAAS). Processing of the samples was performed as previously described [14]. The frozen kidney homogenate samples were dried at $65^{\circ} \mathrm{C}$ for $24 \mathrm{~h}$. Dried samples were then weighed and ashed at $500^{\circ} \mathrm{C}$ for $12 \sim 16 \mathrm{~h}$ in a muffle furnace (Isotemp, Thermo Fisher Scientific, ON, USA). Ashed samples were digested by heating to a slow boil in $1 \mathrm{~mL}$ of concentrated hydrochloric acid $(10 \mathrm{M}$, Thermo Fisher Scientific) and $3 \mathrm{~mL}$ of concentrated nitric acid (6M, Thermo Fisher Scientific) over $4 \sim 6 \mathrm{~h}$. One to two drops of hydrogen peroxide $\left(\mathrm{H}_{2} \mathrm{O}_{2}, 4 \% \mathrm{w} / \mathrm{v}\right)$ were then added, and samples were boiled until only $0.5 \mathrm{~mL}$ of liquid remained. Water was added to concentrated samples $(15 \sim 20 \mathrm{~mL})$, and 


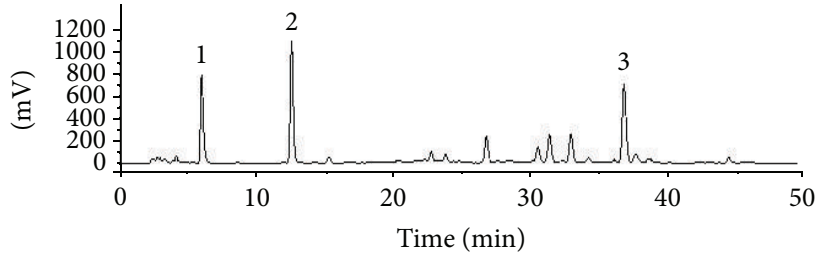

(a)

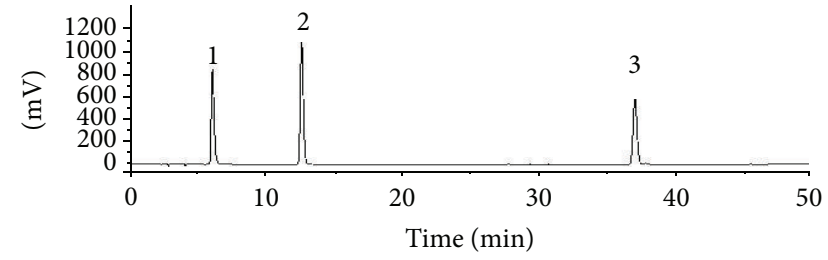

(b)<smiles>O=Cc1ccc(O)c(O)c1</smiles>

(1) Danshensu
(2) Protocatechuic aldehyde<smiles>O=C(/C=C/c1ccc(O)c2c1[C@@H](C(=O)O[C@H](Cc1ccc(O)c(O)c1)C(=O)O)C(c1ccc(O)c(O)c1)O2)O[C@H](Cc1ccc(O)c(O)c1)C(=O)O</smiles>

(3) Salvianolic acid B

(c)

FIGURE 1: HPLC-UV profiles of SM injection, standards, and chemical structures of the active ingredients. HPLC-UV profiles of SM injection (a) and standards (b), and the peaks represented (1) danshensu; (2) protocatechuic aldehyde; (3) salvianolic acid B. (c) The chemical structures of the three active ingredients.

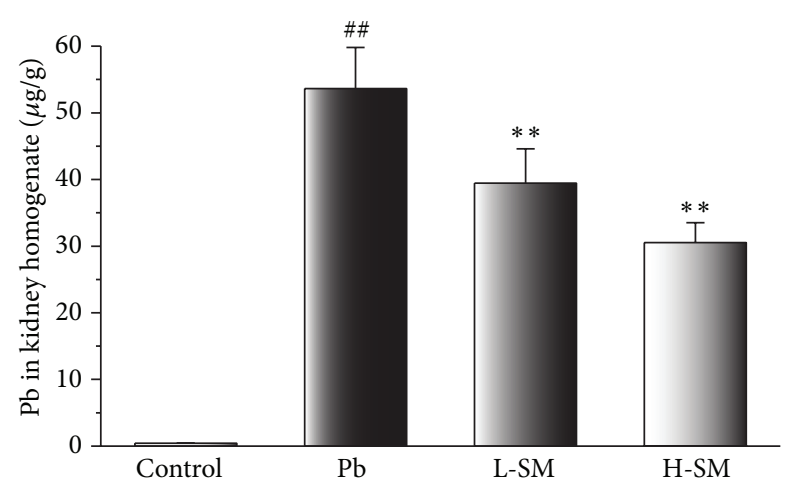

FIGURE 2: Effects of SM injection on concentrations of $\mathrm{Pb}$ in kidney homogenate. Samples were obtained from the control group, $\mathrm{Pb}$ group, L-SM group, and H-SM group. Data are expressed as mean $\pm \mathrm{SD}$ of 20 mice. ${ }^{\# \#} P<0.01$, compared with control group; ${ }^{* *} P<$ 0.01, compared with $\mathrm{Pb}$ group.

absorbance readings were performed at $283.3 \mathrm{~nm}$ using FAAS (Spectra AA-10 Varian Spectrophotometer). Standard curves for $\mathrm{Pb}$ were prepared from commercially available standards (Sigma). BSA 1577A and Tort-2 (National Institute of Standards and Technology, MD, USA) were used as controls for tissue digestion and preparation procedures.

2.6. Hematoxylin and Eosin (H↔E) Examination. Fourmicron sections were cut from paraformaldehyde fixed tissue embedded in paraffin blocks, and then the sections were dewaxed in xylene, rehydrated in descending alcohols, and washed in distilled water. All samples were stained with hematoxylin and eosin (H\&E) for histological observations according to the manufacturer's protocol.
2.7. Assessment of Oxidative Stress and Lipid Peroxidation Indicators. Activities of glutathione peroxidase (GSH-Px) and super oxide dismutase (SOD) and concentration of malondialdehyde (MAD) were detected for assessment of the antioxidant capacity of SM injection. Each kidney tissue sample was weighed to prepare a $10 \%(\mathrm{w} / \mathrm{v})$ buffered homogenate (100 mg tissue $\cdot \mathrm{mL}^{-1}$ of $50 \mathrm{mM}$ phosphate buffer, $\mathrm{pH}$ 7.2). The homogenates were centrifuged, and the supernatant was used for biochemical analyses. The protein concentration of the supernatant was determined by the Lowry method using bovine serum albumin (BSA) as a standard. The activities of GSH-Px and SOD as well as the production of MDA in kidney tissue were analyzed using a spectrophotometer and commercially available kits (Jian Cheng Biological Engineering Institute, Nanjing, China).

2.8. Assessment of Biochemical Indexes. Blood urea nitrogen (BUN) and creatinine (CR) were measured as biochemical indexes for assessment of the renal function. Levels of BUN and CR in serum were evaluated by spectrophotometrybased methods using commercially available kits (Jian Cheng Biological Engineering Institute, Nanjing, China).

\subsection{Terminal Deoxynucleotidyl Transferase-Mediated dUTP} Nick End Labeling (TUNEL) Staining. TUNEL staining was performed using an In Situ Cell Death Detection Kit (Roche Basel, Switzerland) according to the manufacturer's protocol. Briefly, the kidney sections were deparaffinized, dehydrated by a series of successively increasing concentrations of alcohol, washed in distilled water followed by PBS, and deproteinized by proteinase $\mathrm{K}\left(20 \mu \mathrm{g} \cdot \mathrm{mL}^{-1}\right)$ for $30 \mathrm{~min}$ at $37^{\circ} \mathrm{C}$. Then, the sections were rinsed and incubated in the TUNEL reaction mixture. After rinsing, sections were visualized 



FIGURE 3: Effects of SM injection on histopathological changes stained with H\&E. Representative microscopic photographs of kidney stained with $\mathrm{H} \& \mathrm{E}$ (magnification 400x). Compared to the normal morphology of kidneys in the control group, those of mice treated with $\mathrm{Pb}$ acetate displayed marked histological changes in the cortex and remarkable swelling in the proximal tubular epithelial cells, as well as diminishing glomeruli of the kidney. Although SM-treated mice showed some enlargement of proximal tubular epithelial cells, the glomerulus architecture was normal compared with the $\mathrm{Pb}$ group.

using a converter-POD with $0.02 \%$ 3,3-diaminobenzidine (DAB). The sections were counterstained with hematoxylin. The number of tubular cells undergoing apoptosis was counted in three kidney sections of each animal, and the results were expressed as the average number of TUNELpositive cells $/ \mathrm{mm}^{2} \pm \mathrm{SD}$. All counting procedures were performed in a blinded fashion.

2.10. Statistical Analysis. Data were expressed as mean \pm SD. All data were analyzed statistically using analysis of variance (ANOVA), followed by Tukey's multiple comparison test. Statistical significance was considered at $P<0.05$. The Statistical Package for Social Sciences (SPSS, Chicago, IL, USA) for Windows version 15.0 software was used for all analyses.

\section{Results}

3.1. Contents Analysis of SM Injection. The main constituent of SM injection was detected by HPLC-UV (Figures 1(a) and $1(b))$. By calculation, the actual concentrations of the three active ingredients were danshensu $2.15 \mathrm{mg} \cdot \mathrm{mL}^{-1}$, protocatechuic aldehyde $0.44 \mathrm{mg} \cdot \mathrm{mL}^{-1}$, and salvianolic acid B $1.01 \mathrm{mg} \cdot \mathrm{mL}^{-1}$. Furthermore, the chemical structure is shown in Figure 1(c).
3.2. Effects of SM Injection on Body Weight and Kidney Coefficients. The changes of body weight and kidney coefficients are shown in Table 1. The body weight gain of $\mathrm{Pb}$-exposed mice was lower than that of the control group $(P<0.05)$. The body weights were decreased but the kidney weights were increased in the $\mathrm{Pb}$ group because the $\mathrm{Pb}$ accumulated in the kidney and injured the structure and function of the kidney. By the SM injection treatment, the body weight of mice in both L-SM and H-SM groups was higher and the kidney coefficients were lower than the $\mathrm{Pb}$ group (both $P<0.05$ ).

3.3. Effects of SM Injection on Concentrations of Tissues $P b$. As shown in Figure 2, the i.p. injection of $\mathrm{Pb}$ acetate resulted in a dramatic increase of tissue $\mathrm{Pb}$ concentrations. By contrast, SM treatment could significantly decrease the elevated level of $\mathrm{Pb}$ in the kidney homogenate $(P<0.01)$. This phenomenon explains that the increase of kidney coefficient is due to a lot of lead deposition in the kidney and SM could reduce the accumulation.

3.4. Morphological Changes Observed by H\&E Staining. In the $\mathrm{Pb}$ treated mice, pathology slices showed the swelled renal tubule epitheliums and the atrophied glomerulus (Figure 3(b)). Although mice in the L-SM and H-SM treatment groups (Figures 3(c) and 3(d)) showed some enlargement of 


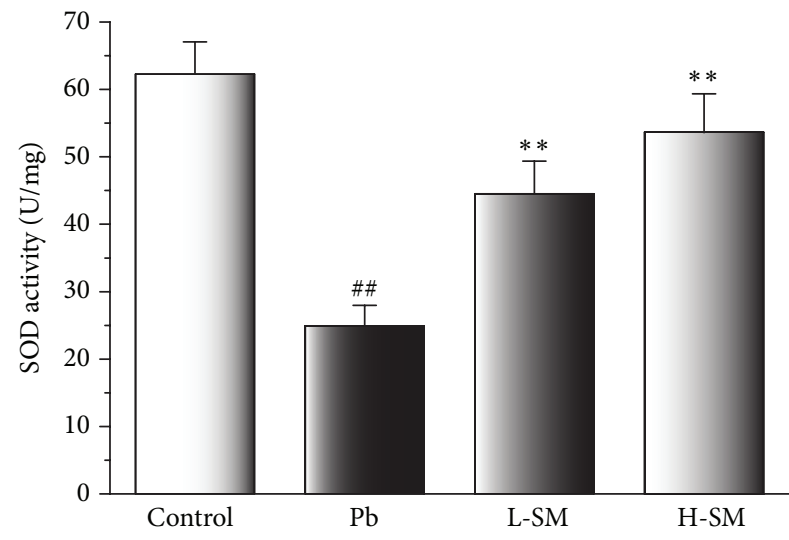

(a)



(b)

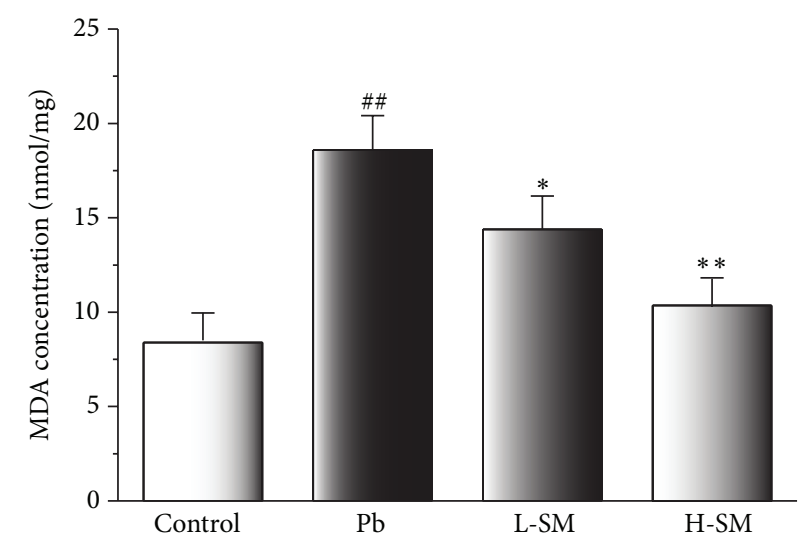

(c)

FIGURE 4: Effects of SM injection on activities of SOD, GSH-Px, and on the concentrations of MDA. Samples were obtained from the kidney homogenate of the experimental mice. Data are expressed as mean $\pm \mathrm{SD}$ of 20 mice. ${ }^{\# \#} P<0.01$, compared with control group; ${ }^{*} P<0.05$, compared with $\mathrm{Pb}$ group; ${ }^{* *} \mathrm{P}<0.01$, compared with $\mathrm{Pb}$ group.

TABLE 1: Effects of SM injection on body weight and kidney coefficients.

\begin{tabular}{lccccc}
\hline Groups & $n$ & IBW $(\mathrm{g})$ & FBW $(\mathrm{g})$ & KW $(\mathrm{g})$ & KC $(\%)$ \\
\hline Control & 20 & $20.28 \pm 2.16$ & $29.17 \pm 2.56$ & $0.31 \pm 0.06$ & $1.09 \pm 0.25$ \\
Pb & 20 & $20.72 \pm 2.23$ & $22.94 \pm 3.07^{\#}$ & $0.37 \pm 0.07$ & $1.78 \pm 0.21^{\#}$ \\
L-SM & 20 & $20.65 \pm 1.72$ & $25.36 \pm 2.23^{*}$ & $0.32 \pm 0.05$ & $1.26 \pm 0.16^{*}$ \\
H-SM & 20 & $19.99 \pm 2.14$ & $26.25 \pm 3.21^{*}$ & $0.31 \pm 0.08$ & $1.18 \pm 0.14^{*}$ \\
\hline
\end{tabular}

IBW: initial body weight; FBW: final body weight; KW: kidney weight; KC: kidney coefficient.

Data are expressed as mean \pm SD. ${ }^{\#} P<0.05$ compared with control group; ${ }^{*} P<0.05$ compared with $\mathrm{Pb}$ group.

the renal tubule epitheliums, the glomerulus's architecture was normal compared with the $\mathrm{Pb}$ group (Figure $3(\mathrm{a})$ ). The results indicated that excessive lead exposure can affect the structure and function of the kidney and the SM injection could ameliorate the injury of the kidney.

3.5. Effects of SM Injection on Tissue SOD, GSH-Px, and MDA. Compared to the control group, activities of SOD and GSH$\mathrm{Px}$ were dramatically decreased in the $\mathrm{Pb}$ group $(P<0.01)$, while the activities of SOD and GSH-Px in the L-SM and $\mathrm{H}-\mathrm{SM}$ groups increased $(P<0.01)$ (Figures $4(\mathrm{a})$ and $4(\mathrm{~b})$ ). Furthermore, the concentration of lipid peroxidative product MDA was significantly enhanced in the $\mathrm{Pb}$ group compared with the control group $(P<0.01)$, while L-SM and H-SM treatment markedly reduced the concentration of MDA $(P<$ 0.01 and $P<0.05$ ) (Figure $4(\mathrm{c})$ ). These results demonstrated that SM treatment could protect against lipid peroxidation in the kidney induced by $\mathrm{Pb}$ exposure and the effect of high dose was better than the low dose.

3.6. Effects of SM Injection on Serum BUN and CR. Compared with control mice, BUN and CR levels were notably elevated in the $\mathrm{Pb}$-exposed animals $(P<0.01)$; meanwhile, serum BUN and CR levels were clearly lower in the SM-treated groups compared with the $\mathrm{Pb}$ group $(P<0.01)$ (Figure 5$)$. These results indicated that SM injection could ameliorate the diminished renal function induced by $\mathrm{Pb}$ exposure. 


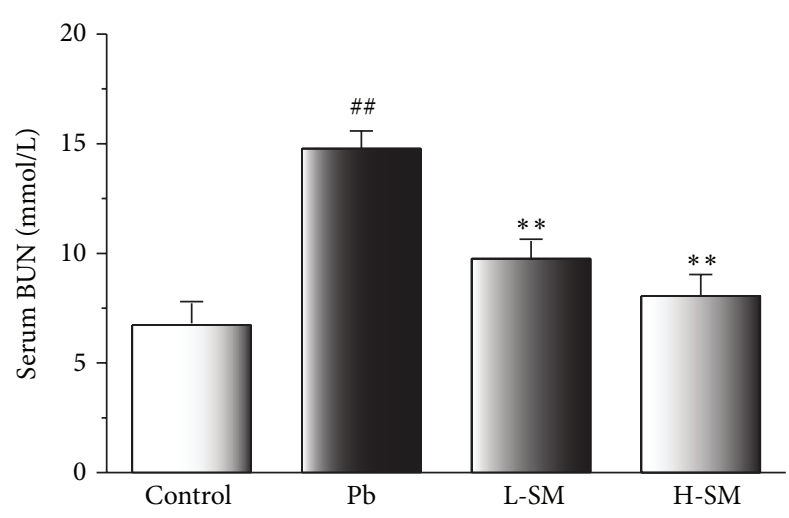

(a)

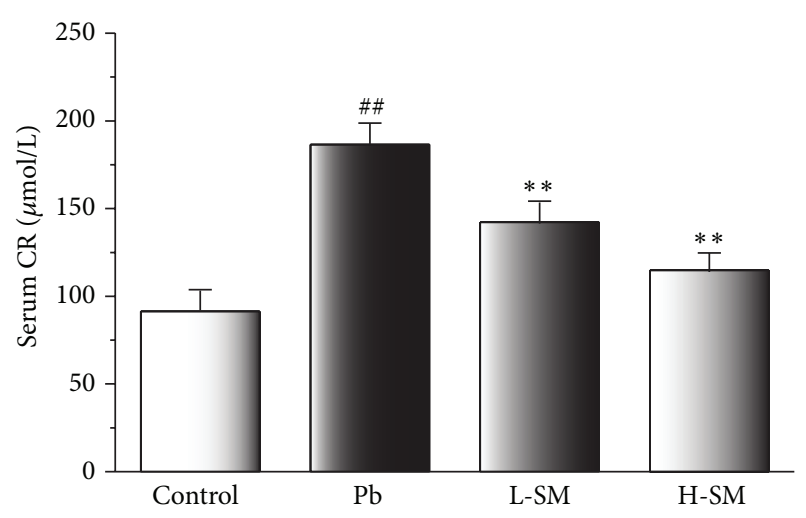

(b)

FIGURE 5: Effects of SM injection on serum concentrations of BUN and CR. Samples were obtained from the control group, Pb group, L-SM group, and H-SM group. Data are expressed as mean \pm SD of 20 mice. ${ }^{\# \#} P<0.01$, compared with control group; ${ }^{* *} P<0.01$, compared with $\mathrm{Pb}$ group.

3.7. Effects of SM Injection on Renal Apoptosis. The extent of tubular cell apoptosis remained at a constantly low level in the control mice, while of TUNEL-positive cells was dramatically raised in the $\mathrm{Pb}$ group and the proportion was decreased to some extent in L-SM and H-SM groups (Figure 6). These results demonstrated that SM injection treatment could suppress the level of renal apoptosis induced by $\mathrm{Pb}$ exposure.

\section{Discussion}

Lead $(\mathrm{Pb})$ is one of the ubiquitous heavy metal poisons in the environment. The pathogenesis of $\mathrm{Pb}$ exposure is multifaceted, including the interruption of enzyme activity and protein function by directing binding to the sulfhydryl group, induction of free radicals, depletion of antioxidant reserves, and the competitive inhibition of mineral absorption and disturbance of homeostasis [15]. The kidney serves as a major organ of $\mathrm{Pb}$ excretion and accumulation and a sensitive target organ for $\mathrm{Pb}$ exposure. In recent studies, $\mathrm{Pb}$ has been shown to inhibit the activities of antioxidant enzymes, including glutathione peroxidase (GSH-Px), catalase, and super oxide dismutase (SOD) [16]. In this study, we have shown that mechanisms of $\mathrm{Pb}$-induced kidney injury are related to increase in production of reactive oxygen species (ROS) and induction of oxidative stress and apoptosis.

SM injection containing bioactive compounds, such as salvianic acid A, protocatechuic aldehyde, and salvianolic acid B (Figure 1), has been used in clinical applications for years with no serious adverse reactions reported [3]. Some of the deleterious effects of $\mathrm{Pb}$ have been attributed to induction of oxidative stress, and $\mathrm{Pb}$ is known to disrupt the prooxidant/antioxidant balance of tissues that leads to biochemical and physiological disturbances [17]. As the commonly used Chinese herbal medicine SM can scavenge oxygen free radicals and inhibit lipid peroxidation, we hypothesized that it may be an effective treatment for kidney injury and therefore investigated its protective effects against renal damage induced by $\mathrm{Pb}$ exposure in mice.
In this investigation, the results demonstrated that SM injection could decrease the kidney coefficient (Table 1), reduce $\mathrm{Pb}$ accumulation (Figure 2), and ameliorate the histopathological changes in the kidney of $\mathrm{Pb}$-exposed mice. Recent pharmacological studies have shown that salvianolic acid B could possibly facilitate the repair of tubular epithelial structures and the regression of renal fibrosis in injured kidney [18]. Furthermore, SM injection could inhibit the lipid peroxidation and decrease renal cell necrosis, thereby protecting the kidney from injury induced by $\mathrm{Pb}$ exposure. $\mathrm{As} \mathrm{Pb}$ dosedependently accumulated in the mice, mostly in the kidney, the excessive $\mathrm{Pb}$ was clearly demonstrated to reduce body growth as well as increasing the kidney weight. However, the kidney coefficient was significantly decreased in all treated animals, indicating that the negative effects of $\mathrm{Pb}$ exposure in mice could be alleviated by $\mathrm{SM}$ injection administration.

$\mathrm{Pb}$-induced toxicity was observed in the glomeruli and proximal tubules of the kidneys in the $\mathrm{Pb}$ group mice (Figure 3). Morphological changes indicated that SM treatment ameliorated the swelling in the renal tubules and the glomerular atrophy induced by $\mathrm{Pb}$ acetate. Danshensu has been demonstrated to inhibit calcium channels in vascular smooth muscle cells, which suggests that SM prevents the entry of $\mathrm{Pb}$ into the kidney tissue via negative effects on calcium channels [19].

Among the various pathogenic mechanisms of renal injury, oxidative stress alters glomerular structure and function mainly due to the effect of reactive oxygen species (ROS) on mesangial and endothelial cells [20]. Accumulating evidence shows that $\mathrm{Pb}$ causes oxidative stress by inducing the generation of ROS, which includs hydroxyl, superoxide radicals, and $\mathrm{H}_{2} \mathrm{O}_{2}$ [21]. $\mathrm{Pb}$ is not able to induce the production of free radicals directly, but it does influence indirectly the processes of lipid peroxidation, which may lead to tissue damage [22]. MDA, the main product of lipid peroxide that can seriously damage the cell membrane, is used as an indicator of cellular membrane damage caused by ROS [23]. SOD and GSH-Px are major enzymatic antioxidants that 

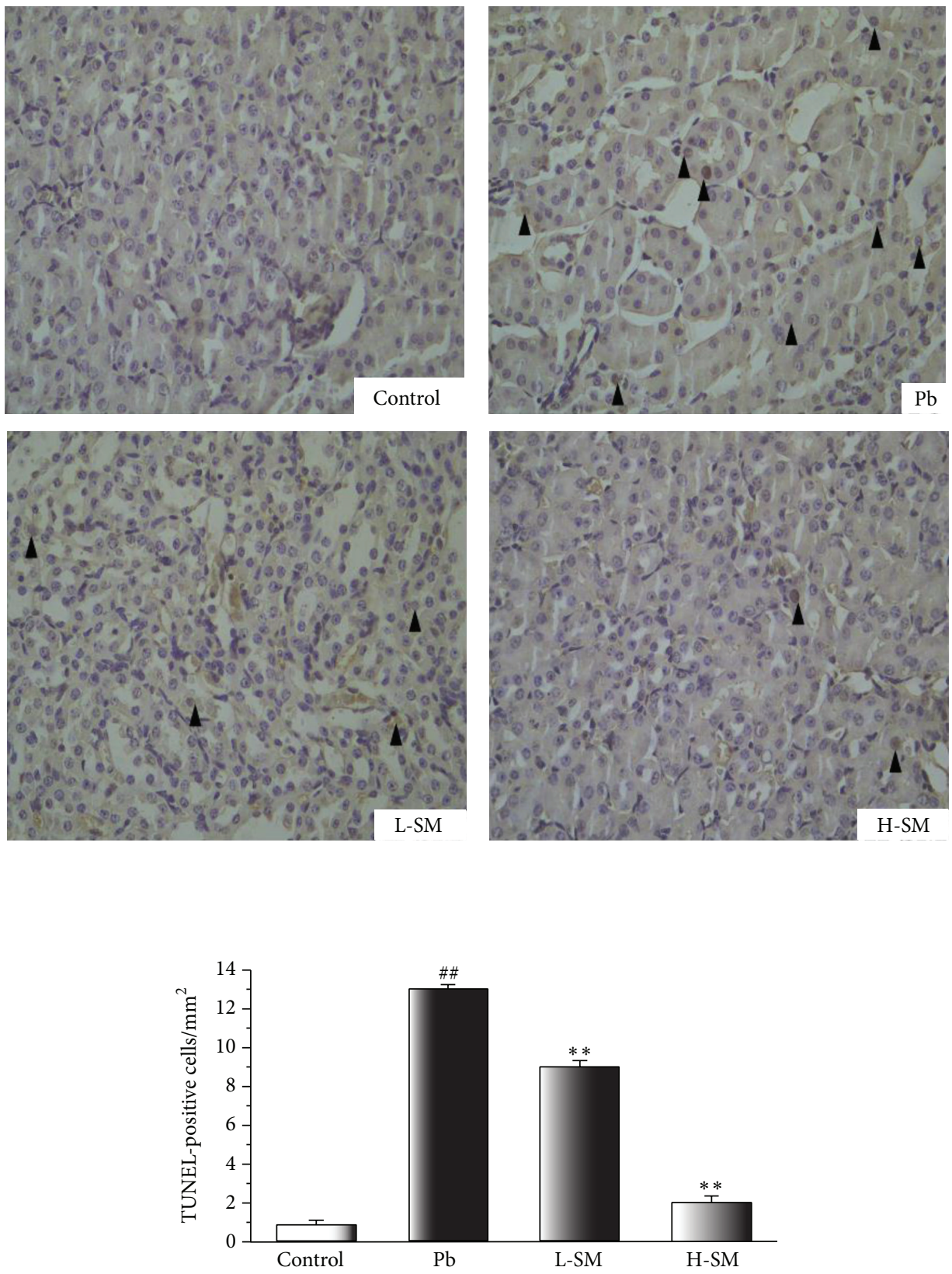

FIGURE 6: Effects of SM injection on the apoptosis of mouse kidneys. Representative microscopic photographs of TUNEL-stained kidney sections were shown (magnification 400x). Very few tubular and interstitial cells were TUNEL-positive in the control group. The number of apoptotic cells markedly increased with lead acetate injection. Both L-SM and H-SM treatments significantly decreased the number of TUNEL-positive cells in the kidney; the arrow heads pointed out the apoptotic cell nucleus. The histogram showed the relative proportion of TUNEL-positive cells in the kidney of mice. Data are expressed as mean \pm SD of 20 mice. ${ }^{\# \#} P<0.01$, compared with control group; ${ }^{* *} P<0.01$, compared with $\mathrm{Pb}$ group.

scavenge harmful ROS, and SOD also provides the first line of defense against free radicals by making toxic superoxide into less toxic $\mathrm{H}_{2} \mathrm{O}_{2}$ [24]. These antioxidant enzymes are potential targets for $\mathrm{Pb}$ toxicity [25]. With the increase in lipid peroxide levels in the body, activities of GSH-Px and SOD change accordingly. In this study, amounts of $\mathrm{Pb}$ accumulated in the kidney escalated the lipid peroxidation (increased MDA concentration) and oxidative stress (lowered SOD and GSHPx activities $)(P<0.01$, Figure 4$)$. With low or high doses of SM injection treatments, animals exhibited lower levels of MDA and higher levels of SOD and GSH-Px than the $\mathrm{Pb}$ group mice (both $P<0.01$ ). Thus, we concluded that SM injection significantly increased the renal antioxidant status by decreasing the content of MDA and increasing the activities of GSH-Px and SOD. The antioxidant properties of SM injection appeared to underlie the mechanism of protective effects against kidney injury.

The kidney has long been recognized as a critical target organ of $\mathrm{Pb}$ toxicity. The serum levels of BUN and CR can be interpreted as indices of renal dysfunction. The results 
showed that serum levels of BUN and CR dramatically increased (both $P<0.01$ ) (Figure 5) in the $\mathrm{Pb}$ group. The increased levels of BUN and CR in the serum reflected deterioration of renal function. Importantly, we found that SM injection could significantly attenuate the elevation of serum BUN and CR levels, indicating that it effectively protected against kidney dysfunction.

Renal cell apoptosis was examined by TUNEL staining and a large number of apoptotic cells were found in the mice treated with $\mathrm{Pb}$ (Figure 6). SM injection administration effectively decreased the cellular apoptosis induced by $\mathrm{Pb}$ exposure in the kidney. Oxidative stress has been found to mediate age-associated renal cell injury and cell death, particularly apoptosis [25]. Furthermore, protocatechuic aldehyde has been reported to protect against TNF- $\alpha$-induced endothelial cell injury by stimulating free radical scavenging activity and has been demonstrated to induce apoptotic cell death by inhibiting the ornithine decarboxylase activity [26].

In conclusion, this study demonstrated multitargeted protective effects of $\mathrm{SM}$ injection on renal damage in $\mathrm{Pb}$ exposed mice by reducing the $\mathrm{Pb}$ accumulated in the kidney, suppressing lipid peroxidation, and inhibiting renal apoptosis. These findings provided preliminary experimental support of SM injection as a therapeutic drug for $\mathrm{Pb}$ poisoning diseases. As SM injection has a complex chemical composition and multiple pharmacological functions, a greater understanding of the mechanisms of SM injection may offer better strategies to protect against kidney dysfunction induced by $\mathrm{Pb}$ associated toxicity.

$\begin{array}{ll}\text { Abbreviations } \\ \text { BUN: } & \text { Blood urea nitrogen } \\ \text { CR: } & \text { Creatinine } \\ \text { GSH-Px: } & \text { Glutathione peroxidase } \\ \text { HPLC-UV: } & \text { High performance liquid chromatography } \\ & \text { with ultraviolet } \\ \text { H-SM: } & \text { High-dose Salvia miltiorrhiza } \\ \text { H\&E: } & \text { Hematoxylin and eosin } \\ \text { i.p.: } & \text { Intraperitoneal } \\ \text { L-SM: } & \text { Low-dose Salvia miltiorrhiza } \\ \text { MDA: } & \text { Malondialdehyde } \\ \text { SOD: } & \text { Superoxide dismutase } \\ \text { TUNEL: } & \text { Terminal deoxynucleotidyl } \\ & \text { transferase-mediated dUTP nick end } \\ & \text { labeling. }\end{array}$

\section{Conflict of Interests}

The authors declare that there is no conflict of interests regarding the publication of this paper.

\section{Authors' Contribution}

Lei Li and Yuanyuan Zhang contributed equally to this work.

\section{Acknowledgments}

This work was supported by the Nature Fund of Hebei Province, China (no. C2011206025), the Fund of Hebei
Science and Technology Bureau (no. 10276105D-2), and the Fund of National College Students Innovation Training Project (no. 201210089004).

\section{References}

[1] A. Cardenas, H. Roels, A. M. Bernard et al., "Markers of early renal changes induced by industrial pollutants. II. Application to workers exposed to lead," British Journal of Industrial Medicine, vol. 50, no. 1, pp. 28-36, 1993.

[2] S. J. S. Flora and V. Pachauri, "Chelation in metal intoxication," International Journal of Environmental Research and Public Health, vol. 7, no. 7, pp. 2745-2788, 2010.

[3] L. Zhou, Z. Zuo, and M. S. S. Chow, "Danshen: an overview of its chemistry, pharmacology, pharmacokinetics, and clinical use," Journal of Clinical Pharmacology, vol. 45, no. 12, pp. 1345-1359, 2005.

[4] D. G. Kang, H. Oh, E. J. Sohn et al., "Lithospermic acid B isolated from Salvia miltiorrhiza ameliorates ischemia/ reperfusion-induced renal injury in rats," Life Sciences, vol. 75, no. 15, pp. 1801-1816, 2004.

[5] J. P. Zhang, Y. Y. Zhang, Y. Zhang et al., "Salvia miltiorrhiza (Danshen) injection ameliorates iron overload-induced cardiac damage in mice," Planta Medica, vol. 79, no. 9, pp. 744-752, 2013.

[6] Y. Gao, N. Wang, Y. Zhang et al., "Mechanism of protective effects of Danshen against iron overload-induced injury in mice," Journal of Ethnopharmacology, vol. 145, no. 1, pp. 254260, 2013.

[7] Y. Zhang, Y. Xie, Y. Gao et al., "Multitargeted inhibition of hepatic fibrosis in chronic iron-overloaded mice by Salvia miltiorrhiza," Journal of Ethnopharmacology, vol. 148, no. 2, pp. 671-681, 2013.

[8] S. Guan, J. Ma, Y. Zhang et al., "Danshen (Salvia miltiorrhiza) injection suppresses kidney injury induced by iron overload in mice," PLoS ONE, vol. 8, no. 9, Article ID e74318, 2013.

[9] L. E. Kerper and P. M. Hinkle, "Cellular uptake of lead is activated by depletion of intracellular calcium stores," The Journal of Biological Chemistry, vol. 272, no. 13, pp. 8346-8352, 1997.

[10] J. L. Tomsig and J. B. Suszkiw, "Permeation of $\mathrm{Pb}^{2+}$ through calcium channels: fura-2 measurements of voltage- and dihydropyridine-sensitive $\mathrm{Pb}^{2+}$ entry in isolated bovine chromaffin cells," Biochimica et Biophysica Acta, vol. 1069, no. 2, pp. 197200, 1991.

[11] J. Zhang, H. Cao, Y. Zhang et al., "Nephroprotective effect of calcium channel blockers against toxicity of lead exposure in mice," Toxicology Letters, vol. 218, no. 3, pp. 273-280, 2013.

[12] C. Cao, Q. Xia, X. Zhang, W. Xu, H. Jiang, and J. Chen, "Salvia miltiorrhiza attenuates the changes in contraction and intracellular calcium induced by anoxia and reoxygenation in rat cardiomyocytes," Life Sciences, vol. 72, no. 22, pp. 2451-2463, 2003.

[13] F. F. Y. Lam, J. H. K. Yeung, Y. W. Kwan, K. M. Chan, and P. M. Y. Or, "Salvianolic acid B, an aqueous component of danshen (Salvia miltiorrhiza), relaxes rat coronary artery by inhibition of calcium channels," European Journal of Pharmacology, vol. 553, no. 1-3, pp. 240-245, 2006.

[14] S. Selander and K. Cramér, "Determination of lead in blood by atomic absorption spectrophotometry," British Journal of Industrial Medicine, vol. 25, no. 3, pp. 209-213, 1968. 
[15] L. Patrick, "Lead toxicity, a review of the literature, part I: exposure, evaluation, and treatment," Alternative Medicine Review, vol. 11, no. 1, pp. 2-22, 2006.

[16] C. M. Bolin, R. Basha, D. Cox et al., "Exposure to lead and the developmental origin of oxidative DNA damage in the aging brain," The FASEB Journal, vol. 20, no. 6, pp. 788-790, 2006.

[17] R. Neal, K. Cooper, H. Gurer, and N. Ercal, "Effects of Nacetylcysteine and 2,3-dimercaptosuccinic acid on lead induced oxidative stress in rat lenses," Toxicology, vol. 130, no. 2-3, pp. 167-174, 1998.

[18] G. Yao, L. Xu, X. Wu, L. Xu, J. Yang, and H. Chen, "Preventive effects of salvianolic acid $b$ on transforming growth factor- $\beta 1$ induced epithelial-to-mesenchymal transition of human kidney cells," Biological and Pharmaceutical Bulletin, vol. 32, no. 5, pp. 882-886, 2009.

[19] F. F. Y. Lam, J. H. K. Yeung, K. M. Chan, and P. M. Or, "Relaxant effects of danshen aqueous extract and its constituent danshensu on rat coronary artery are mediated by inhibition of calcium channels," Vascular Pharmacology, vol. 46, no. 4, pp. 271-277, 2007.

[20] S. Thamilselvan and S. R. Khan, "Oxalate and calcium oxalate crystals are injurious to renal epithelial cells: results of in vivo and in vitro studies," Journal of Nephrology, vol. 11, supplement 1, no. 1, pp. 66-69, 1998.

[21] M. Yurekli, M. Esrefoglu, M. I. Doĝru, A. Doĝru, M. Gul, and M. Whidden, "Adrenomedullin reduces antioxidant defense system and enhances kidney tissue damage in cadmium and lead exposed rats," Environmental Toxicology, vol. 24, no. 3, pp. 279-286, 2009.

[22] O. Ademuyiwa, R. Agarwal, R. Chandra, and J. R. Behari, "Leadinduced phospholipidosis and cholesterogenesis in rat tissues," Chemico-Biological Interactions, vol. 179, no. 2-3, pp. 314-320, 2009.

[23] P. C. Sharpe, W. Liu, K. K. M. Yue et al., "Glucose-induced oxidative stress in vascular contractile cells: comparison of aortic smooth muscle cells and retinal pericytes," Diabetes, vol. 47, no. 5, pp. 801-809, 1998.

[24] P. Geisser, "Iron therapy and oxidative stress," Metal-Based Drugs, vol. 4, no. 3, pp. 137-152, 1997.

[25] P. Hsu and Y. L. Guo, "Antioxidant nutrients and lead toxicity," Toxicology, vol. 180, no. 1, pp. 33-44, 2002.

[26] Z. Chang, E. Gebru, S. Lee et al., "In vitro antioxidant and antiinflammatory activities of protocatechualdehyde isolated from Phellinus gilvus," Journal of Nutritional Science and Vitaminology, vol. 57, no. 1, pp. 118-122, 2011. 

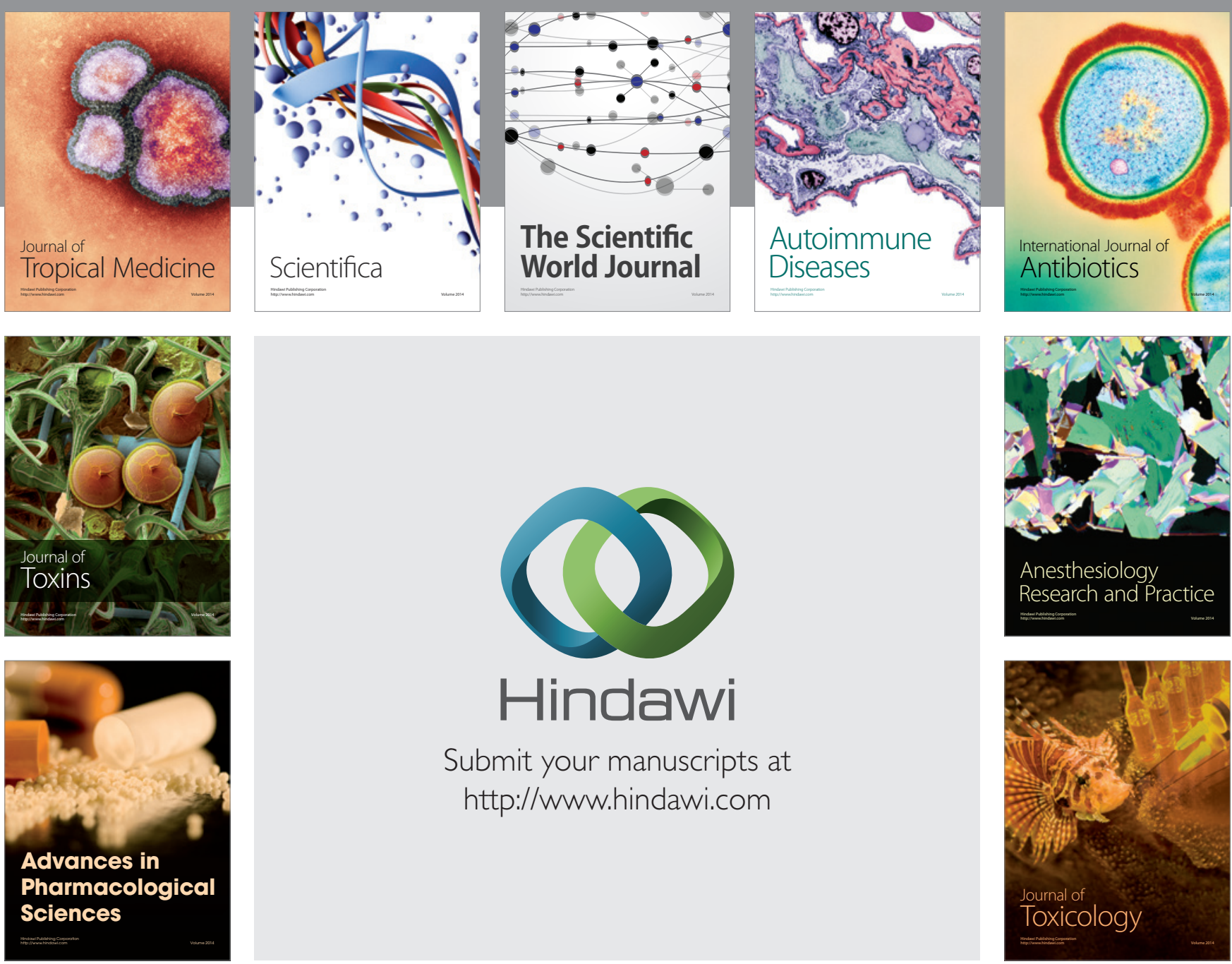

\section{Hindawi}

Submit your manuscripts at

http://www.hindawi.com
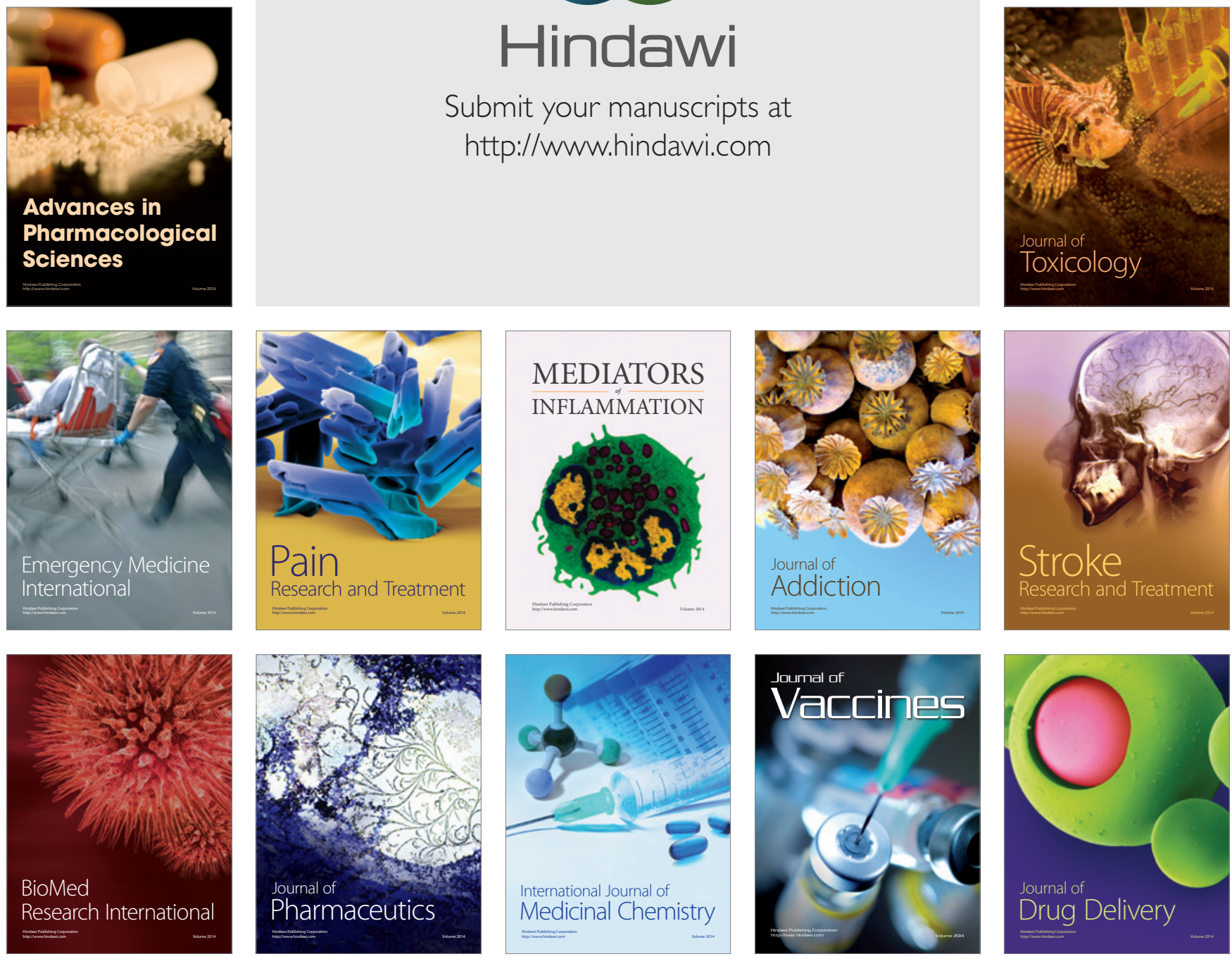